\section{(2) OPEN ACCESS}

\title{
Cumulative health deficits, $A P O E$ genotype, and risk for later-life mild cognitive impairment and dementia
}

\author{
David D Ward (ㄱ, 1,2 Lindsay M K Wallace, ${ }^{3}$ Kenneth Rockwood (1) 1,2
}

\begin{abstract}
- Additional material is published online only. To view, please visit the journal online (http://dx.doi.org/10.1136/ innp-2020-324081).
\end{abstract}

${ }^{1}$ Geriatric Medicine Research, Centre for Health Care of the Elderly, Nova Scotia Health Authority, Halifax, Nova Scotia, Canada

2Department of Medicine, Dalhousie University, Halifax Nova Scotia, Canada

${ }^{3}$ Faculty of Graduate Studies, Dalhousie University, Halifax, Nova Scotia, Canada

\section{Correspondence to}

Professor Kenneth Rockwood, Geriatric Medicine Research, Centre for Health Care of the Elderly, Nova Scotia Health Authority, Halifax, Canada; kenneth.rockwood@dal.ca

Received 1 June 2020 Revised 12 September 2020 Accepted 19 October 2020 Published Online First 13 November 2020

\author{
ABSTRACT \\ Objective To determine whether health-deficit \\ accumulation is associated with the risks of mild \\ cognitive impairment $(\mathrm{MCI})$ and dementia independently \\ of $A P O E$ genotype.
}

Methods A frailty index was calculated using the deficit-accumulation approach in participants aged 50 years and older from the National Alzheimer's Coordinating Center. Cognitive status was determined by clinical evaluation. Using multistate transition models, we assessed the extent to which an increasing degree of frailty affected the probabilities of transitioning between not cognitively impaired ( $\mathrm{NCl}$, $\mathrm{MCl}$, and dementia.

Results Participants ( $n=14490)$ had a mean age of 72.2 years ( $S D=8.9$ years; range $=50-103$ years). Among those $\mathrm{NCl}$ at baseline ( $n=9773$ ), each 0.1 increment increase in the frailty index was associated with a higher risk of developing $\mathrm{MCl}$ and a higher risk of progressing to dementia. Among those with $\mathrm{MCl}$ at baseline $(n=4717)$, higher frailty was associated with a higher risk of progressing to dementia, a lower probability of being reclassified as $\mathrm{NCl}$, and a higher likelihood of returning to $\mathrm{MCl}$ in those that were reclassified as $\mathrm{NCl}$. These risk effects were present and similar in both carriers and noncarriers of the APOE $\varepsilon 4$ allele.

Conclusion Among older Americans, health-deficit accumulation affects the likelihood of progressive cognitive impairment and the likelihood of cognitive improvement independently of a strong genetic risk factor for dementia. Frailty represents an important risk factor for cognitive dysfunction and a marker of potential prognostic value.

\section{INTRODUCTION}

Even though mild cognitive impairment (MCI) commonly represents an intermediate clinical stage prior to dementia, ${ }^{1}$ it lacks specificity in identifying who will later develop dementia. ${ }^{2} 3$ In many with MCI, cognition does not decline, whereas others are later reclassified as no longer cognitively impaired, ${ }^{4}$ some of whom can later again be classified as having MCI or even dementia. 5 Accurately identifying which older individuals are at an increased risk of progressive cognitive decline and dementia can help elucidate disease mechanisms and improve clinical and public health practice.

Many age-related cardiovascular, neuropsychiatric, and other health deficits are more common among those who develop MCI and dementia than among those who do not. ${ }^{67}$ Although biological pathways link some individual dementia risk factors to neurodegenerative processes, the breadth of known risk factors indicates that poor general health may play a role in dementia development. In support, healthy lifestyles are associated with a lower dementia risk in later life. ${ }^{89}$ Examining the accumulation of age-related health deficits, or measuring the degree of frailty, ${ }^{10}$ also affords insight into how general health affects dementia risk. Frailty is an age-related health state that is associated with an increased risk of adverse events, dementia, and death. ${ }^{11-13}$ Measuring each individual's degree of frailty may help identify those individuals without cognitive impairment most likely to develop MCI, ${ }^{14}$ and those with MCI most likely to progress to dementia. ${ }^{15}$ Critically, despite recent recommendations in support of preventing and mitigating frailty ${ }^{16}$ — chiefly through lifestyle interventions that overlap with those proposed for dementia-it is not yet known whether frailty plays an equal role in dementia development in relation to a genetic predisposition to dementia. Recent evidence is mixed about whether a healthy lifestyle reduces dementia risk in those at high genetic risk. ${ }^{89}$ For better prevention and treatment, ${ }^{17}$ we must better understand these relationships.

Using data from the National Alzheimer's Coordinating Center (NACC), we pursued four objectives: (1) detail the dynamic nature of cognitive functioning by calculating the likelihood of transitions between cognitive states in both directions over a 12-month period; (2) assess the extent to which an increasing degree of frailty is associated with changing cognitive-state transition probabilities; (3) provide context for any observed associations of frailty and transition probabilities by calculating and comparing the corresponding associations of other risk factors (chronological age, education level, and the APOE \&4 allele); (4) determine whether frailty acts on risk of MCI and dementia independently or through interaction with genetic risk (APOE $\varepsilon 4$ carrier status).

\section{METHOD}

\section{Participants}

Data came from the NACC (http://www.alz.washington.edu), contributed by Alzheimer's Disease Research Centers (ADRCs) located across the USA and funded by the National Institute on Aging. Participants included those who had MCI and Alzheimer's disease and related disorders, as well as a subset of healthy volunteers. Since 2005 , these ADRCs have collected data using a prospective, standardised and longitudinal clinical evaluation of subjects, which forms the uniform data set (UDS). For the UDS, baseline and subsequent follow-up 


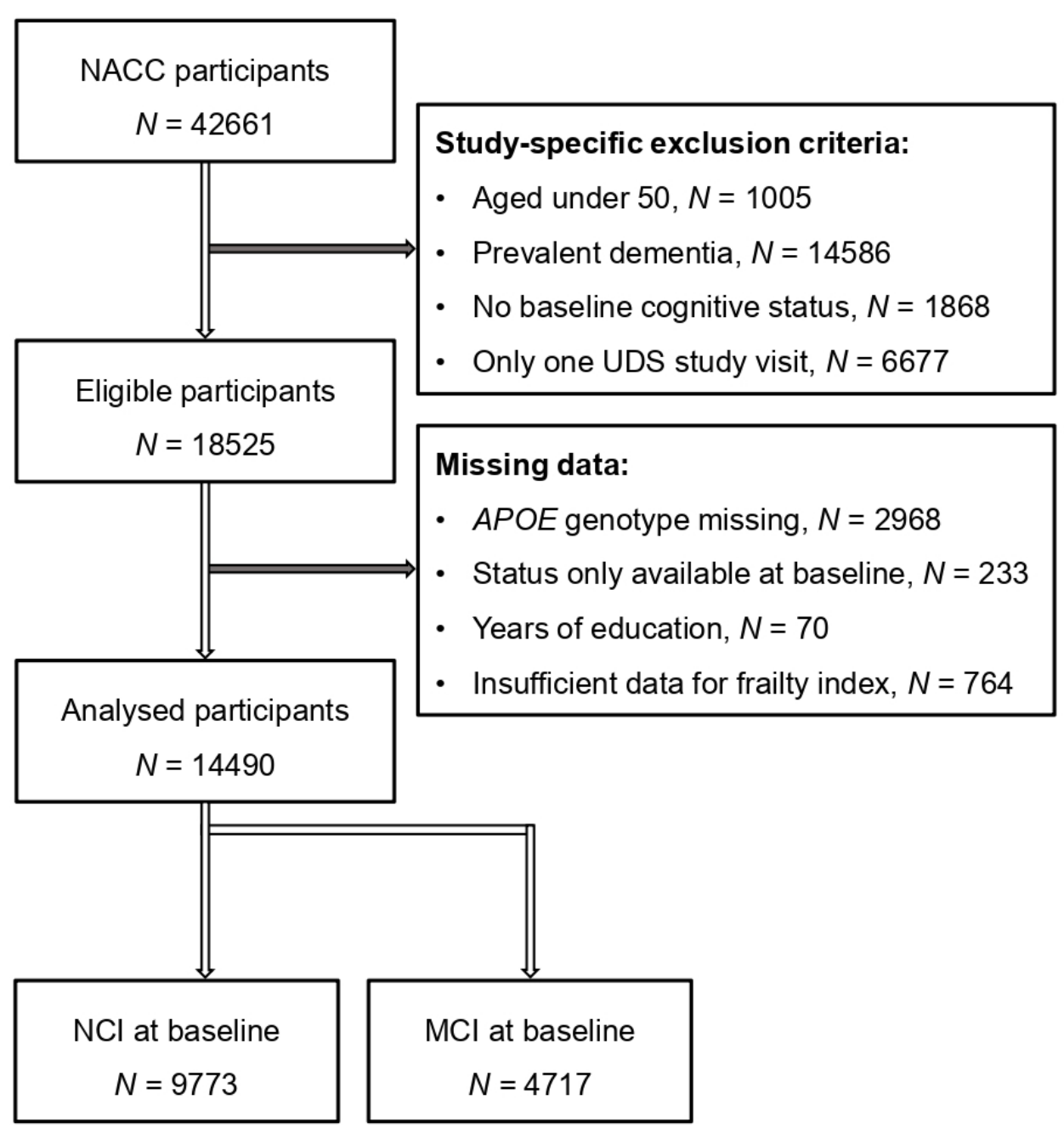

Figure 1 Study sample flow chart. MCI, mild cognitive impairment; NACC, National Alzheimer's Coordinating Center; NCl, not cognitively impaired; UDS, Uniform Data Set.

data (approximately annually) were collected by clinicians and clinic personnel, and contains deidentified information on participants' sociodemographics, neurological exam findings, functional status, neuropsychological test results, clinical diagnoses and APOE genotype.

Here, the sample was drawn from UDS visits conducted between September 2005 and February 2020 (figure 1). We included participants aged 50 years or older, with available data on cognitive status at the initial UDS visit, and at least two recorded UDS visits. Some participants were missing data on covariates or had insufficient data to calculate a baseline frailty index and were excluded. Apart from the Mini-Mental State Examination score being a half-point lower in those with data missing, and their median frailty index scores being 0.02 points higher, participants who were eligible but were excluded due to missing data were similar to those included (table 1).

\section{Data availability}

Participants provided informed consent at the ADRC prior to data collection. These data are publicly available through the NACC.

\section{Clinical classification}

Each participant underwent a clinical evaluation at each UDS visit. Diagnoses of cognitive status were made by either a consensus team or a single physician, depending on the specific ADRC's protocol. Although diagnostic criteria for dementia were not provided by the NACC to each ADRC until 2015, it is assumed that most ADRCs were using DSM-IV (or similar) criteria. We coded a cognitive status variable with three levels: not cognitively impaired (NCI), MCI and dementia. Individuals were categorised as NCI if the clinician(s) determined them to have normal cognition and behaviour, as MCI if the clinician(s) diagnosed one of any MCI subtypes (amnestic or non-amnestic, single domain or multiple domain), or as dementia if they met the criteria for all-cause dementia.

\section{Frailty index}

We calculated a frailty index score for each participant using their baseline assessment. The frailty index is a health-state measure, incorporating information from multiple physiological systems, and closely reflects an individual's risk for adverse health events and mortality independently of chronological age. ${ }^{10} \mathrm{~A}$ higher frailty index score indicates accumulation of more age-related health deficits, yet does not include any information on chronological age, thereby approximating biological age. ${ }^{18}$ The health variables included in a frailty index are routinely collected clinical data such as symptoms, signs, disabilities and diseases that meet standard criteria. ${ }^{19}$ The frailty index score represents the 
Table 1 Characteristics of the sample at study entry

\begin{tabular}{|c|c|c|c|c|}
\hline \multirow[b]{2}{*}{ Characteristic } & \multirow[b]{2}{*}{ Participants with missing data } & \multirow[b]{2}{*}{ Total analysed sample } & \multicolumn{2}{|c|}{ Analysed participants } \\
\hline & & & $\mathrm{NCl}$ at baseline & $\mathrm{MCl}$ at baseline \\
\hline $\mathrm{N}(\%)$ & $4035(22)$ & $14490(78)$ & $9773(67)$ & $4717(33)$ \\
\hline Age, years, mean $(S D)$ & $73.0(8.9)$ & $72.2(8.9)$ & $71.7(9.0)$ & $73.3(8.6)$ \\
\hline \multicolumn{5}{|l|}{ Sex, N (\%) } \\
\hline Men & $1550(38)$ & $5865(40)$ & $3407(35)$ & $2458(52)$ \\
\hline Women & $2485(62)$ & $8625(60)$ & $6366(65)$ & 2259 (48) \\
\hline \multicolumn{5}{|l|}{ Race, N (\%) } \\
\hline White & $2982(74)$ & $11806(81)$ & 7914 (81) & $3892(83)$ \\
\hline Black & $680(17)$ & $1776(12)$ & $1269(13)$ & 507 (11) \\
\hline Asian & $137(3)$ & $343(2)$ & $209(2)$ & $134(3)$ \\
\hline Multiracial/other & $236(6)$ & $565(4)$ & $381(4)$ & $184(4)$ \\
\hline Education, years, mean $(S D)$ & $15.6(3.2)$ & $15.7(3.1)$ & $15.9(2.9)$ & $15.4(3.3)$ \\
\hline Follow-up data, years, mean (SD) & $3.0(2.9)$ & $5.3(3.5)$ & $5.8(3.6)$ & $4.4(3.0)$ \\
\hline MMSE score, mean $(S D)$ & $28.0(2.2)$ & $28.4(2.0)$ & $28.9(1.4)$ & $27.1(2.5)$ \\
\hline \multicolumn{5}{|l|}{ Frailty index score } \\
\hline Median (IQR) & $0.11(0.07-0.16)$ & $0.09(0.05-0.14)$ & $0.08(0.04-0.11)$ & $0.13(0.09-0.20)$ \\
\hline Range & $0.00-0.53$ & $0.00-0.56$ & $0.00-0.51$ & $0.00-0.56$ \\
\hline \multicolumn{5}{|l|}{$A P O E \& 4$ status, $\mathrm{N}(\%)$} \\
\hline$\varepsilon 4$ non-carrier & $513(70)$ & $9459(65)$ & $6801(70)$ & $2658(56)$ \\
\hline$\varepsilon 4$ carrier & $221(30)$ & $5031(35)$ & $2972(30)$ & 2059 (44) \\
\hline
\end{tabular}

As participants were excluded due to missing covariate data (age, sex, education and APOE $\varepsilon 4$ status), the values presented here represent the mean values for participants in which data on those variables were available. Ninety-three missing race values were recoded to 'Multiracial/other' for descriptive purposes. Some proportions may not sum to $100 \%$ due to rounding.

$\mathrm{MCl}$, mild cognitive impairment; MMSE, Mini-Mental State Examination; NCl, not cognitively impaired.

proportion of total health deficits that an individual possesses. For example, a person with 10 of 40 assessed health deficits has a frailty index score of $10 / 40=0.25$, meaning that higher scores on a frailty index indicate worse health. Estimates of an individual's degree of frailty are consistent even when the health variables included in its calculation vary, so long as more than approximately 40 health variables are included. ${ }^{20}$ In calculating a frailty index for NACC participants, we identified and screened 67 candidate health variables from the UDS. After applying standard criteria, ${ }^{19} 45$ health deficits were retained and used to calculate frailty index scores (online supplemental table 1). Health deficits that were closely related to our main outcome of cognitive status were excluded (eg, performance on tests of cognitive functioning). Frailty index scores were multiplied by 10 so that associated HRs could be meaningfully interpreted as the change in risk for a transition per 0.1 increase in the frailty index score, which here is equivalent to an additional four to five deficits. As in recent reports that related frailty and neuropathology, ${ }^{13} 21$ we calculated an additional frailty index for use in sensitivity analyses that excluded 13 additional items with possible association to dementia (online supplemental table 1).

\section{Other risk factors}

Sex and years of education were collected via self-report. Chronological age was calculated from the self-reported month and year of birth. APOE genotyping was reported to the NACC. Age, education level and $A P O E \varepsilon 4$ allelic status were important to our study, given their known associations with risk of MCI and/or dementia. ${ }^{7}$

\section{Statistical analysis}

We first characterised the demographic and clinical characteristics of the analytical sample using summary statistics. We next used the R package 'msm' (Multi-State Markov and
Hidden Markov Models in Continuous Time) V.1.6.8 $8^{22}$ to calculate multistate Markov models. For the multistate models, we defined three possible states through which an individual could move (NCI, MCI and dementia) and one absorbing state (death). Given that MCI is often considered a precursor to dementia, we assumed that individuals moved through this state before transitioning to dementia (figure 2). We also assumed that cognitive functioning could decline (forward transitions) and improve (backward transitions). The multistate transition models were conducted separately for participants who were NCI (NCI subsample) and who had MCI (MCI subsample) at initial UDS visit. We first calculated the 12-month transition probabilities between different cognitive states within each subsample prior to entering explanatory variables into the models (objective 1).

We next quantified the associations of the baseline frailty index score, age at baseline (years), sex (men, women), education level (years), and APOE $\varepsilon 4$ allelic status ( $\varepsilon 4$ carrier, $\varepsilon 4$ noncarrier) with the probabilities of transitioning between states (objectives 2 and 3). In each case, the relationship was expressed as a HR and accompanied by $95 \%$ CIs. The HRs for the frailty index score represented the change in risk of transition per 0.1 increase in the frailty index. We also calculated these associations by $A P O E \& 4$ status, and tested differences using an interaction term (objective 4). We conducted three sensitivity analyses: the first repeated the analyses using a frailty index with items that might represent early symptoms of dementia removed from its calculation; the second explored whether any identified associations of frailty and transition probabilities differed between men and women; the third examined the associations of frailty and transition probabilities after removing participants whose race was other than white (in the absence of a sufficient sample size for race subgroup analyses). All statistical models included each covariate (frailty index score, age at baseline, sex, education 


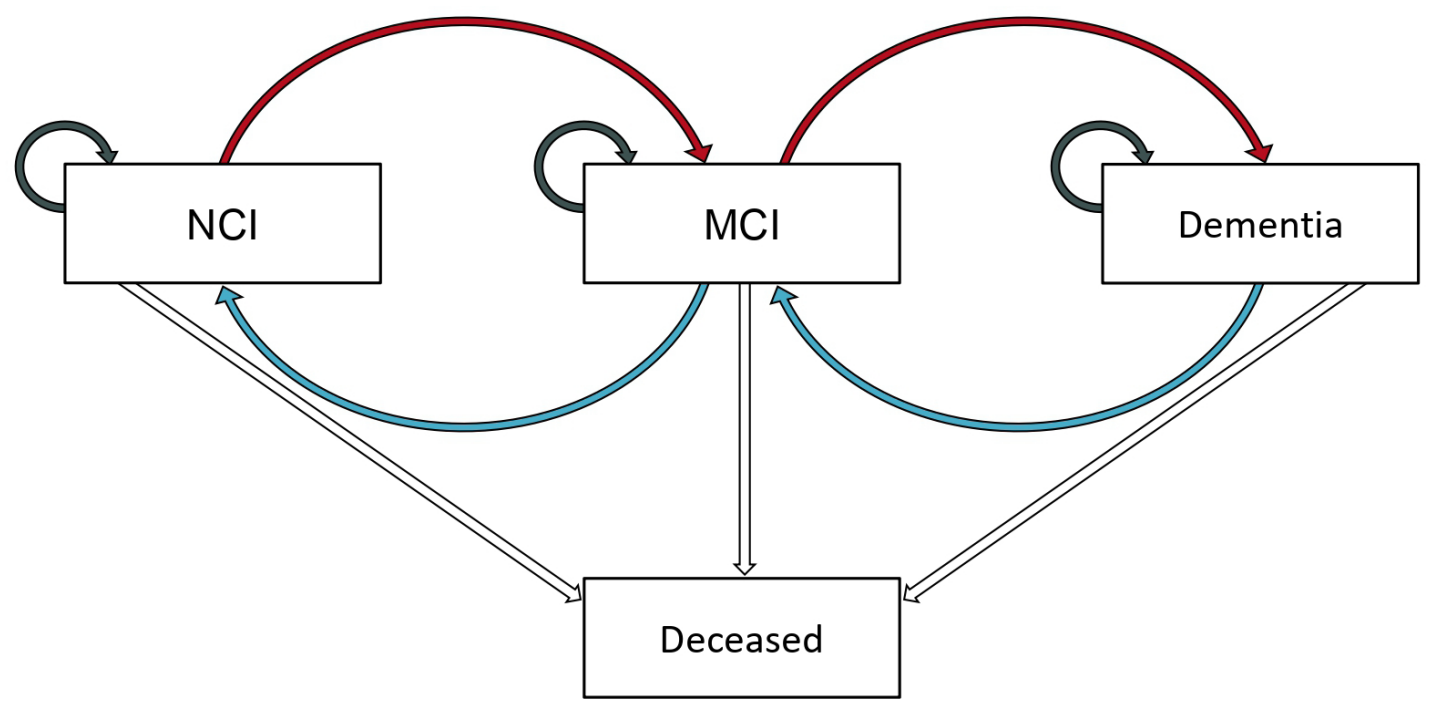

Figure 2 The multistate model. Red arrows indicate transitions to a state of worse cognitive impairment (forward transitions), blue arrows indicate transitions to a state of better cognitive functioning (backward transitions), and grey arrows indicate that the cognitive state was maintained over two successive UDS study visits. $\mathrm{MCl}$, mild cognitive impairment; $\mathrm{NCl}$, not cognitively impaired.

level, and $A P O E \varepsilon 4$ allelic status). We undertook all statistical analyses using R V.3.6.1.

\section{RESULTS}

Sample

Of 18525 eligible participants, 14490 were included in the analytical sample (figure 1) yielding 78635 observations (total observation time $=77204.1$ person years). The analytical sample ranged in age from 50 to 103 years old, and in length of follow-up from 6 months to 14 years (table 1). The distribution of baseline frailty index scores, stratified by subsample, is presented in online supplemental figure 1).

\section{State transitions}

Over 12 months, NCI subsample participants maintained their prior state 43086 times $(90.6 \%)$ and transitioned between states 4491 times (9.4\%), $3086(68.7 \%)$ of which were transitions between cognitive states, with 1405 (31.3\%) transitions to death (objective 1, table 2). MCI subsample participants maintained their prior state 12957 times (73.3\%) and transitioned between states 4729 times (26.7\%), 3590 (75.9\%) of which were transitions between cognitive states, with 1139 (24.1\%) deaths. The substantial proportion of transitions to death for both subsamples highlights the considerable competing risk of death before the onset of dementia. Of the cognitive-state transitions in the NCI subsample, $80.9 \%$ were forward transitions (ie, to a state of worse impairment) and 19.1\% were backward transitions (ie, to a state of better functioning). In the MCI subsample, $70.5 \%$ were forward transitions and $29.5 \%$ were backward transitions.

\section{Frailty and state transitions}

We assessed the direction and strength of relationships between an increasing degree of frailty and the probabilities of cognitivestate transitions (objective 2). In the NCI subsample, we observed a higher degree of frailty (estimated in 0.1 increments) to be associated with a higher risk of transitioning from NCI to MCI and from MCI to dementia (figure 3). In the MCI subsample, we observed a higher degree of frailty to be associated with a lower probability of being reclassified as NCI from MCI, a higher risk of returning to MCI in those who were reclassified as NCI, and a higher risk of progressing to dementia. The direction and statistical significance of relationships were mostly robust in a sensitivity analysis that removed 13 items that might represent early symptoms of dementia from its calculation (online supplemental figure 2). However, frailty was no longer associated at a statistically significant level with the risk of progressing to dementia from MCI in the NCI subsample, or with the risk of returning to MCI from NCI in the MCI subsample. The associations of the frailty index score with transition probabilities did not differ significantly between men and women (online supplemental figure 3) and were similar when participants whose race was other than white were removed from the analytical sample (online supplemental figure 4).

\section{Other risk factors and state transitions}

The HRs and 95\% CIs for the associations of age, sex, years of education, and APOE $\varepsilon 4$ allelic status with each transition are presented alongside the corresponding associations of increasing frailty in online supplemental table 2) (objective 3). Briefly, each

Table 2 Observed transitions and 12-month transition probabilities

\begin{tabular}{|c|c|c|c|c|c|c|c|c|c|c|}
\hline \multirow[b]{2}{*}{ Subsample } & \multicolumn{2}{|c|}{ Forward transitions } & \multicolumn{2}{|c|}{ Backward transitions } & \multicolumn{3}{|c|}{ State maintenance over successive study visits } & \multicolumn{3}{|c|}{ Transitions between states and death } \\
\hline & $\mathrm{NCl}$ to $\mathrm{MCl}$ & $\mathrm{MCl}$ to $\mathrm{DEM}$ & $\mathrm{NCl}$ from $\mathrm{MCl}$ & $\mathrm{MCI}$ from DEM & $\mathrm{NCl}$ & $\mathrm{MCl}$ & DEM & $\mathrm{NCl}$ & $\mathrm{MCl}$ & DEM \\
\hline $\mathrm{NCl}$ at baseline & $2014(4.7 \%)$ & $483(15.1 \%)$ & $542(14.6 \%)$ & $47(4.5 \%)$ & $40197(93.4 \%)$ & $1973(63.8 \%)$ & $916(73.6 \%)$ & $783(1.3 \%)$ & $275(6.5 \%)$ & $347(21.5 \%)$ \\
\hline $\mathrm{MCl}$ at baseline & $341(15.3 \%)$ & $2191(16.9 \%)$ & $925(8.0 \%)$ & $133(2.6 \%)$ & $1535(81.0 \%)$ & $7684(73.4 \%)$ & $3738(84.1 \%)$ & $58(2.0 \%)$ & $280(1.6 \%)$ & $801(13.2 \%)$ \\
\hline
\end{tabular}




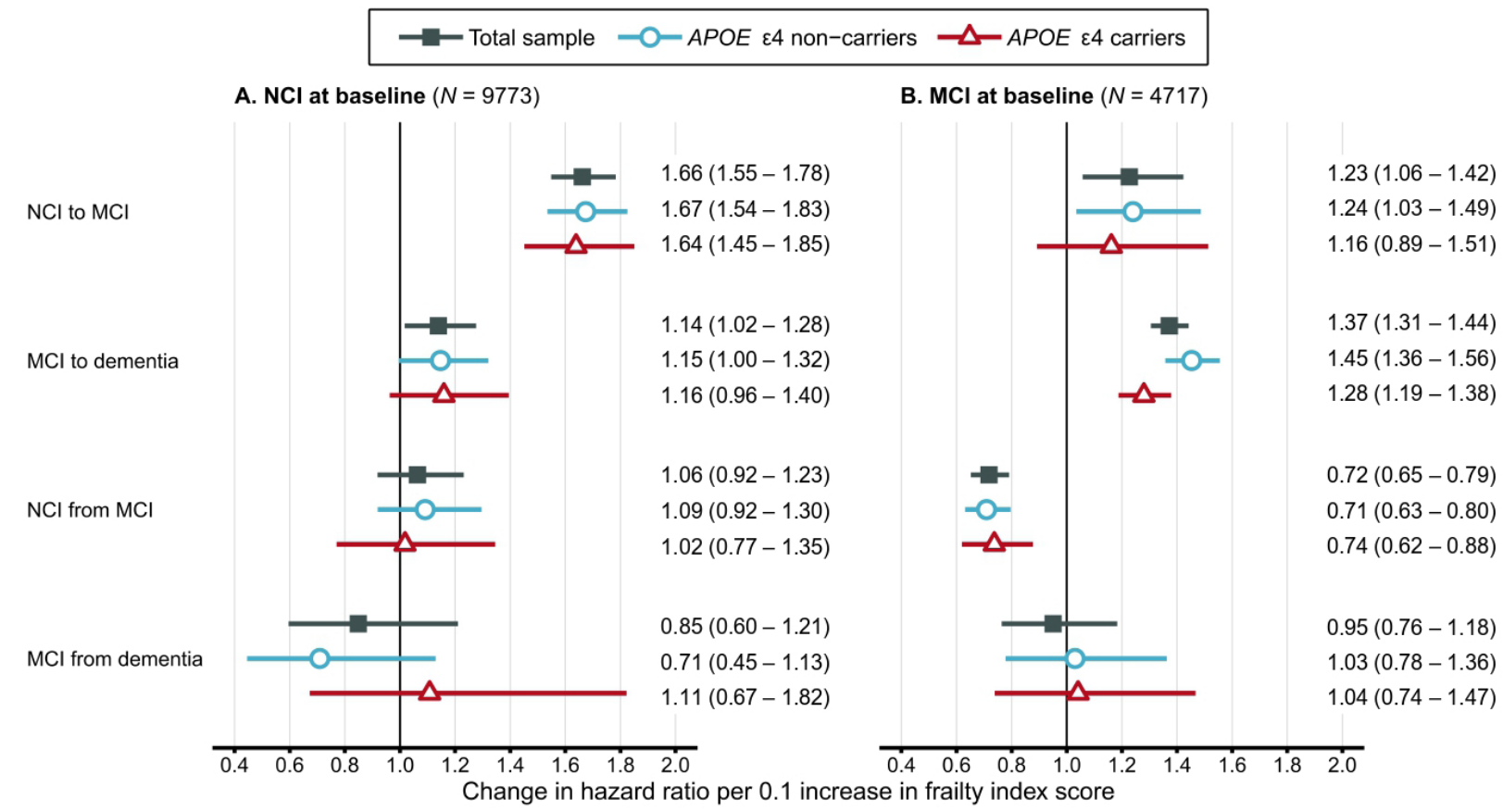

Figure 3 Associations of the frailty index score and the probabilities of cognitive-state transitions for the total subsamples and APOE $\varepsilon 4$ carriers and non-carriers, separately. HRs were calculated from multistate transition models stratified by baseline cognitive status as well as APOE $\varepsilon 4$ carrier status and presented with 95\% Cls. Associations were adjusted for age at baseline, sex, education level and APOE \&4 allelic status. MCl, mild cognitive impairment; $\mathrm{NCl}$, not cognitively impaired.

additional year of age at baseline and carrying APOE $\varepsilon 4$ were both associated with an increased risk of transitioning to states of greater impairment and a lower probability of being reclassified at states of less impairment, although the statistical significance of associations varied by subsample. Each additional year of education was associated with a lower risk of developing MCI in the NCI subsample, and a lower probability of being reclassified as MCI from dementia in the MCI subsample. In the NCI subsample, men had a higher risk of MCI but also a higher probability of being reclassified as NCI from MCI. However, a lower probability of being reclassified as NCI from MCI was observed in men relative to women in the MCI subsample.

\section{Frailty, $A P O E$ and state transitions}

We explored whether the frailty index score and APOE ع4 carrier status exerted independent or interactive effects on cognitive-state transition probabilities (objective 4). In the NCI subsample, no statistically significant interactions were observed between these variables for any transition (figure 3). In the MCI subsample, the association of the frailty index score and the risk of progressing to dementia was significantly weaker in those carrying an $A P O E \& 4$ allele than in non-carriers (interaction $\mathrm{HR}=0.88(95 \%$ CI 0.80 to 0.97$))$ and was robust in an analysis using the sensitivity frailty index. No meaningful differences in these associations were observed when participants whose race was other than white were removed from the analytical sample (online supplemental figure 4).

\section{DISCUSSION}

We observed that cognitive functioning is a dynamic process; both functional decline and functional improvement are common. Greater frailty was associated with greater risk of developing MCI and of progressing to dementia; the additional risk associated with each 0.1 increase in the frailty index score was typically comparable to carrying an APOE $\varepsilon 4$ allele, as well as to a considerable number of additional years of age. These frailty risk effects were present and similar in both carriers and non-carriers of the APOE $\varepsilon 4$ allele. Our results implicate frailty in playing a key role in dementia development by increasing the likelihood of progressive cognitive impairment and reducing the likelihood of cognitive recovery. They also suggest that intervening to prevent or reduce frailty may decrease incident cognitive impairment and dementia even in those carrying an $A P O E$ \&4 allele, a proposition that requires further testing. These findings complement and extend the results of a recent systematic review and meta-analysis that identified the common co-occurrence of physical frailty and cognitive impairment among those individuals most likely to develop dementia, ${ }^{23}$ and support findings from the UK Biobank whereby dementia risk may be modified by general health factors (ie, lifestyle) at any level of genetic risk. ${ }^{8}$

Although older age is often considered to be characterised by functional decline, we observed that functional improvement was common in our sample. These results support work that detailed cognitive change as a dynamic process, ${ }^{45}$ especially at the level of cognitive test scores. ${ }^{24} 25$ We observed annual transition probabilities of $4.7 \%$ for $\mathrm{NCI}$ to $\mathrm{MCI}$ (decline) and $14.6 \%$ for NCI from MCI (improvement) in the NCI subsample. These transition probabilities closely reflect those of a prospective cohort study from Olmsted County, Minnesota, which reported annual transition rates of $6.2 \%$ and $12.3 \%$, respectively, among 1450 participants who were cognitively normal at baseline. ${ }^{26}$ That study also reported approximately 9 more cases of MCI per 1000 person years among men than among women. Similarly, we observed men to have both a $19 \%$ higher risk of developing MCI and a $14 \%$ higher probability of later being reclassified as NCI than women. In our study, frailty also appeared to be more strongly associated with transition probabilities in men relative to in women, although these effects did not reach statistical significance. 
Frailty is non-controversially associated with impairment in later-life cognition. This is the case for changes in cognitive test scores, ${ }^{24} 27$ as well as for the risks of MCI and dementia. ${ }^{11141528}$ Here, we additionally explored whether these associations depended on APOE $\varepsilon 4$ carrier status, quantified sex differences (as did Song et $a l^{11}$ ) rather than solely adjusting for them, and extended this work to a large, mostly clinic-based setting. In the NCI subsample, we observed each 0.1 increment in the frailty index score (equivalent to 4-5 additional health deficits) increased the risk of MCI by $66 \%$. In the MCI subsample, each 0.1 increment in the frailty index score increased the risk of progressing to dementia by $37 \%$ and reduced the probability of being reclassified as NCI by $28 \%$. Our sensitivity analysis indicated that these relationships were robust, although somewhat weaker, after the removal of 13 items (29\% of total considered deficits) that might represent symptoms of dementia from the calculation of the frailty index.

This degree of risk is non-trivial. Consider the corresponding associations of chronological age and the APOE $\varepsilon 4$ allele, each a potent risk, and both of which exerted statistically significant effects on risk of cognitive-state transitions. For the risk of MCI among the NCI subsample, each 0.1 increase in the frailty index score was the equivalent of 11 additional years of age, and an increase in frailty index score of 0.06 (about three additional health deficits) was equal to the additional risk of carrying an $A P O E \& 4$ allele. For the risk of progressing to dementia in the MCI subsample, each 0.1 increase in the frailty index score was the equivalent of 12.3 additional years of age, and an increase in frailty index score of 0.15 (about seven additional health deficits) was equal to the additional risk of carrying an APOE $\varepsilon 4$ allele. Within the same MCI subsample, the probability of being reclassified as NCI was also lower in accordance with increasing frailty. Here, each 0.1 increase in the frailty index score was the equivalent of 14 years of age, and an increase in frailty index score of 0.13 (about six deficits) was equal to the additional risk of carrying an APOE \&4 allele.

Our results bolster the notion that modifiable risk factors offer a pathway to dementia prevention even in those at significant genetic risk for the condition. Recently, a healthy lifestyle was demonstrated to lower dementia risk at any level of polygenic risk for Alzheimer's disease. ${ }^{8}$ Similarly, we have shown here that another multiply determined modifiable factor affects dementia risk independently of the APOE \&4 allele, and even after the onset of clinically significant cognitive impairment (ie, $\mathrm{MCI}$ ). In the MCI subsample, although we observed a weaker effect of frailty on dementia risk in APOE $\varepsilon 4$ carriers than in non-carriers, that difference was small. Here, the MCI subsample had more transitions from MCI to dementia ( $n=2191$ vs $\mathrm{n}=483$ ) and had more APOE $\varepsilon 4$ carriers (44\% vs 30\%), yielding substantially greater power to detect this interaction. Although our findings indicate that frailty is an important risk factor for MCI and dementia regardless of APOE, interventions aimed at preventing or reducing frailty may show even larger benefits in those without an APOE $\varepsilon 4$ allele. In contrast to our findings, it is worth noting a recent report that found a stronger relationship between frailty and memory decline in APOE $\varepsilon 4$ carriers only, ${ }^{29}$ but there the authors explored these associations in a smaller population-based sample and used continuous measures of cognitive functioning rather than clinical diagnoses.

Frailty, observed here exerting a detrimental influence on later-life cognitive trajectories at multiple transition points represents an attractive intervention target for reducing the population incidence of cognitive impairment. Given the multiply determined nature of frailty, multidomain interventions are the most likely to produce meaningful and clinically relevant improvements in degree of frailty. ${ }^{30}$ Three recent large-scale multidomain interventions notably benefited later-life cognition through reductions in the prevalence of medical and lifestylerelated risk factors. ${ }^{31}$ It is possible that frailty acted as a partial mediator of the intervention-related cognitive benefits, but this warrants further research. ${ }^{32}$ Aside from lifestyle interventions, pharmacological treatments that target biological ageing may hold significant promise, but their development is ongoing. ${ }^{33}$

Our study has limitations. First, the volunteers who contribute towards the NACC are not representative of the USA population, and our analytical sample therefore likely included more incident cases of dementia and had a higher average degree of frailty than would normally be observed. Even so, our transition probabilities closely reflected those of another populationbased prospective cohort study. ${ }^{26}$ Second, for interpretability and generalisability of results to population health, we collapsed all MCI subtypes into our MCI state. It is, however, possible that frailty exerts differential effects on risk of converting to dementia (or on the probability of being reclassified as NCI) for different MCI subtypes. Third, as a minority of our sample was Black (12\%) and less common state transitions were observed in small number within these participants, we were unable to include race as an explanatory variable in our models. Blacks have approximately twice the dementia risk of whites, ${ }^{34}$ a proportion of which is caused by the higher prevalence of both modifiable health deficits and the APOE $\varepsilon 4$ allele. ${ }^{35}$ Our results were consistent when compared with those yielded from models in which only whites were analysed, indicating that the inclusion of NACC participants of all races did not substantially alter our findings. Future research on how these associations may present differently as a function of race is imperative and a priority for our future work.

We conclude that frailty is a key risk factor for age-related cognitive dysfunction and dementia, representing both a target for interventions aimed at the prevention of age-related cognitive impairment and a possible prognostic marker among those who have MCI. This work supports an emerging conceptualisation of late-onset dementia as a complex outcome of ageing that often is intimately related to an individual's general health, as well as genetic risk factors.

Twitter David D Ward @David_D_Ward, Lindsay M K Wallace @WallaceLindsay and Kenneth Rockwood @Krockdoc

Acknowledgements This work was supported by a Canadian Institutes of Health Research Project Grant (PJT-156114). LMKW receives doctoral study support from the Canadian Institutes of Health Research (GSD-154170). KR receives career support from the Dalhousie Medical Research Foundation as the Kathryn Allen Weldon Professor of Alzheimer Research, and research support from the Canadian Institutes of Health Research, the Nova Scotia Health Research Foundation, the Canadian Frailty Network, the Capital Health Research Fund and the Fountain Family Innovation Fund of QEII Health Sciences Centre Foundation. The NACC database is funded by NIA/NIH Grant U01 AG016976. NACC data are contributed by the NIAfunded ADCs: P30 AG019610 (PI Eric Reiman, MD), P30 AG013846 (PI Neil Kowall, MD), P30 AG062428-01 (PI James Leverenz, MD) P50 AG008702 (PI Scott Small, MD), P50 AG025688 (PI Allan Levey, MD, PhD), P50 AG047266 (PI Todd Golde, MD, PhD), P30 AG010133 (PI Andrew Saykin, PsyD), P50 AG005146 (PI Marilyn Albert, PhD), P30 AG062421-01 (PI Bradley Hyman, MD, PhD), P30 AG062422-01 (PI Ronald Petersen, MD, PhD), P50 AG005138 (PI Mary Sano, PhD), P30 AG008051 (PI Thomas Wisniewski, MD), P30 AG013854 (PI Robert Vassar, PhD), P30 AG008017 (PI Jeffrey Kaye, MD), P30 AG010161 (PI David Bennett, MD), P50 AG047366 (PI Victor Henderson, MD, MS), P30 AG010129 (PI Charles DeCarli, MD), P50 AG016573 (PI Frank LaFerla, PhD), P30 AG062429-01(PI James Brewer, MD, PhD), P50 AG023501 (PI Bruce Miller, MD), P30 AG035982 (PI Russell Swerdlow, MD), P30 AG028383 (PI Linda Van Eldik, PhD), P30 AG053760 (PI Henry Paulson, MD, PhD), P30 AG010124 (PI John Trojanowski, MD, PhD), P50 AG005133 (PI Oscar Lopez, MD), P50 AG005142 (PI Helena Chui, MD), P30 AG012300 (PI Roger Rosenberg, MD), P30 AG049638 (PI Suzanne Craft, PhD), P50 AG005136 (PI Thomas Grabowski, MD), P30 
AG062715-01 (PI Sanjay Asthana, MD, FRCP), P50 AG005681 (PI John Morris, MD), P50 AG047270 (PI Stephen Strittmatter, MD, PhD).

Contributors DDW and KR designed and conceptualised the study; DDW analysed the data, wrote the first draft, and revised all drafts; DDW, LMKW, and KR interpreted the data; LMKR and KR reviewed all drafts; KR supervised the project.

Funding The authors have not declared a specific grant for this research from any funding agency in the public, commercial or not-for-profit sectors.

Competing interests KR has asserted copyright of the Clinical Frailty Scale through Dalhousie University's Industry, Liaison, and Innovation Office. Use is free for education, research, and not-for-profit health care. Users agree not to change or commercialize the scale. In addition to academic and hospital appointments, KR is co-founder of DGI Clinical, which in the last five years has contracts with pharma and device manufacturers (Biogen, Shire, Hollister, Novartis, Nutricia, Roche, Takeda) on individualized outcome measurement. In 2017 he attended an advisory board meeting with Lundbeck on dementia, and in 2020 chaired a Scientific Workshop \& Technical Review Panel on frailty for the Singapore National Research Foundation. Otherwise any personal fees are for invited guest lectures, rounds and academic symposia, received directly from event organizers, for presentations on frailty. He is Associate Director of the Canadian Consortium on Neurodegenerataion in Aging, which is funded by the Canadian Institutes for Health Research, the Alzheimer Society of Canada, and several other charities.

Patient consent for publication Not required.

Ethics approval The NACC is approved by the University of Washington Institutional Review Board.

Provenance and peer review Not commissioned; externally peer reviewed.

Data availability statement Data are available in a public, open access repository. Data from the National Alzheimer's Coordinating Center (NACC) are available from the publicly available NACC Uniform Data Set database (https://www. alz.washington.edu/).

Supplemental material This content has been supplied by the author(s). It has not been vetted by BMJ Publishing Group Limited (BMJ) and may not have been peer-reviewed. Any opinions or recommendations discussed are solely those of the author(s) and are not endorsed by BMJ. BMJ disclaims all liability and responsibility arising from any reliance placed on the content. Where the content includes any translated material, BMJ does not warrant the accuracy and reliability of the translations (including but not limited to local regulations, clinical guidelines, terminology, drug names and drug dosages), and is not responsible for any error and/or omissions arising from translation and adaptation or otherwise.

Open access This is an open access article distributed in accordance with the Creative Commons Attribution Non Commercial (CC BY-NC 4.0) license, which permits others to distribute, remix, adapt, build upon this work non-commercially, and license their derivative works on different terms, provided the original work is properly cited, appropriate credit is given, any changes made indicated, and the use is non-commercial. See: http://creativecommons.org/licenses/by-nc/4.0/.

ORCID iDs

David D Ward http://orcid.org/0000-0001-5476-9526

Kenneth Rockwood http://orcid.org/0000-0002-6674-995X

\section{REFERENCES}

1 Petersen RC, Caracciolo B, Brayne C, et al. Mild cognitive impairment: a concept in evolution. J Intern Med 2014;275:214-28.

2 Ganguli M, Dodge $\mathrm{HH}$, Shen $\mathrm{C}$, et al. Mild cognitive impairment, amnestic type: an epidemiologic study. Neurology 2004;63:115-21.

3 Lopez OL, Becker JT, Chang Y-F, et al. Incidence of mild cognitive impairment in the Pittsburgh cardiovascular health Study-Cognition study. Neurology 2012;79:1599-606

4 Ganguli M, Snitz BE, Saxton JA, et al. Outcomes of mild cognitive impairment by definition: a population study. Arch Neurol 2011;68:761-7.

5 Roberts RO, Knopman DS, Mielke MM, et al. Higher risk of progression to dementia in mild cognitive impairment cases who revert to normal. Neurology 2014;82:317-25.

6 Knopman DS, Gottesman RF, Sharrett AR, et al. Midlife vascular risk factors and midlife cognitive status in relation to prevalence of mild cognitive impairment and dementia in later life: the Atherosclerosis risk in Communities study. Alzheimers Dement 2018;14:1406-15.
7 Livingston G, Sommerlad A, Orgeta V, et al. Dementia prevention, intervention, and care. Lancet 2017;390:2673-734.

8 Lourida I, Hannon E, Littlejohns TJ, et al. Association of lifestyle and genetic risk with incidence of dementia. JAMA 2019:322:430-7.

9 Licher S, Ahmad S, Karamujić-Čomić H, et al. Genetic predisposition, modifiablerisk-factor profile and long-term dementia risk in the general population. Nat Med 2019;25:1364-9.

10 Rockwood K, Howlett SE. Age-Related deficit accumulation and the diseases of ageing. Mech Ageing Dev 2019;180:107-16.

11 Song X, Mitnitski A, Rockwood K. Age-Related deficit accumulation and the risk of late-life dementia. Alzheimers Res Ther 2014;6:54.

12 Clegg A, Young J, lliffe S, et al. Frailty in elderly people. Lancet 2013;381:752-62.

13 Wallace LMK, Theou O, Godin J, et al. Investigation of frailty as a moderator of the relationship between neuropathology and dementia in Alzheimer's disease: a crosssectional analysis of data from the rush memory and aging project. Lancet Neurol 2019;18:177-84

14 Boyle PA, Buchman AS, Wilson RS, et al. Physical frailty is associated with incident mild cognitive impairment in community-based older persons. J Am Geriatr Soc 2010:58:248-55.

15 Trebbastoni A, Canevelli M, D'Antonio F, et al. The Impact of Frailty on the Risk of Conversion from Mild Cognitive Impairment to Alzheimer's Disease: Evidences from a 5-Year Observational Study. Front Med 2017;4:178.

16 Ismail Z, Black SE, Camicioli R, et al. Recommendations of the 5th Canadian consensus conference on the diagnosis and treatment of dementia. Alzheimers Dement 2020;16:1182-95.

17 Rockwood K, Wallace LMK, Davis DH. Genetic predisposition and modifiable risks for late-life dementia. Nat Med 2019;25:1331-2.

18 Li X, Ploner A, Wang Y, et al. Longitudinal trajectories, correlations and mortality associations of nine biological ages across 20-years follow-up. Elife 2020;9:e51507.

19 Searle SD, Mitnitski A, Gahbauer EA, et al. A standard procedure for creating a frailty index. BMC Geriatr 2008;8:24.

20 Rockwood K, Mitnitski A. Frailty in relation to the accumulation of deficits. J Gerontol A Biol Sci Med Sci 2007;62:722-7.

21 Wallace LMK, Theou O, Darvesh S, et al. Neuropathological burden and the degree of frailty in relation to global cognition and dementia. Neurology 2020;95:1-11.

22 Jackson CH. Multi-State Models for Panel Data: The msm Package for R. J Stat Softw 2011;38:1-28

23 Grande G, Haaksma ML, Rizzuto D, et al. Co-Occurrence of cognitive impairment and physical frailty, and incidence of dementia: systematic review and meta-analysis. Neurosci Biobehav Rev 2019:107:96-103.

24 Armstrong JJ, Mitnitski A, Andrew MK, et al. Cumulative impact of health deficits, social vulnerabilities, and protective factors on cognitive dynamics in late life: a multistate modeling approach. Alzheimers Res Ther 2015;7:38.

25 Mitnitski $A B$, Fallah $N$, Dean $C B$, et al. A multi-state model for the analysis of changes in cognitive scores over a fixed time interval. Stat Methods Med Res 2014;23:244-56

26 Roberts RO, Geda YE, Knopman DS, et al. The incidence of MCI differs by subtype and is higher in men: the Mayo clinic study of aging. Neurology 2012;78:342-51.

27 Mitnitski A, Fallah N, Rockwood MRH, et al. Transitions in cognitive status in relation to frailty in older adults: a comparison of three frailty measures. J Nutr Health Aging 2011:15:863-7.

28 Song X, Mitnitski A, Rockwood K. Nontraditional risk factors combine to predict Alzheimer disease and dementia. Neurology 2011;77:227-34.

29 Thibeau S, McDermott K, McFall GP, et al. Frailty effects on non-demented cognitive trajectories are moderated by sex and Alzheimer's genetic risk. Alzheimers Res Ther 2019;11:55

30 Apóstolo J, Cooke R, Bobrowicz-Campos E, et al. Effectiveness of interventions to prevent pre-frailty and frailty progression in older adults: a systematic review. JBI Database System Rev Implement Rep 2018;16:140-232.

31 Kivipelto M, Mangialasche F, Ngandu T. Lifestyle interventions to prevent cognitive impairment, dementia and Alzheimer disease. Nat Rev Neurol 2018;14:653-66.

32 Livingston G, Huntley J, Sommerlad A, et al. Dementia prevention, intervention, and care: 2020 report of the Lancet Commission. Lancet 2020:396:413-46.

33 Kennedy BK, Pennypacker JK. Drugs that modulate aging: the promising yet difficult path ahead. Trans/ Res 2014;163:456-65.

34 Weuve J, Barnes LL, Mendes de Leon CF, et al. Cognitive aging in black and white Americans: cognition, cognitive decline, and incidence of Alzheimer disease dementia. Epidemiology 2018;29:151-9.

35 Barnes LL, Bennett DA. Alzheimer's Disease In African Americans: Risk Factors And Challenges For The Future. Health Aff 2014;33:580-6. 